Proceedings of the Edinburgh Mathematical Society (2006) 49, 101-113 (C)

DOI:10.1017/S001309150400063X Printed in the United Kingdom

\title{
SPECTRUM OF THE LAPLACIAN OF AN ASYMMETRIC FRACTAL GRAPH
}

\author{
JONATHAN JORDAN \\ Department of Probability and Statistics, University of Sheffield, Hounsfield Road, \\ Sheffield S3 7RH, UK (jonathan.jordan@sheffield.ac.uk)
}

(Received 8 July 2004)

\begin{abstract}
We consider a simple self-similar sequence of graphs that does not satisfy the symmetry conditions that imply the existence of a spectral decimation property for the eigenvalues of the graph Laplacians. We show that, for this particular sequence, a very similar property to spectral decimation exists, and we obtain a complete description of the spectra of the graphs in the sequence.
\end{abstract}

Keywords: fractal graph; Laplacian; spectral decimation

2000 Mathematics subject classification: Primary 28A80

\section{Introduction and definitions}

\subsection{Introduction}

Many self-similar graphs, and related fractals, display a property known as spectral decimation: that the spectrum of the Laplacian can be described in terms of the iteration of a rational function $f$. Eigenvalues $\lambda$ of the Laplacian at a given stage of the construction are related to eigenvalues $\mu$ of the Laplacian at the following stage of the construction by the relationship

$$
\lambda=f(\mu)
$$

where $f$ is a rational function on $\mathbb{R}$, unless $\mu$ is a member of a small exceptional set, $\mathcal{E}$. This was first observed for the specific case of the Sierpiński gasket graph in [8], and this was given a rigorous mathematical treatment in $[\mathbf{4}, \mathbf{1 1}, \mathbf{1 2}]$. In the case of the Sierpiński gasket, using our definition of the Laplacian (see $\S 1.3$ ), the function $f(\mu)=\mu(5-4 \mu)$ and the exceptional set is $\left\{\frac{1}{2}, \frac{5}{4}, \frac{3}{2}\right\}$.

A generalization of spectral decimation to a much larger class of self-similar graphs, including the Vicsek set graph, appears in [7], in which a symmetry condition is developed which, if satisfied, ensures that spectral decimation applies to the graph. Each self-similar graph in this class has a function $f$ and exceptional set $\mathcal{E}$ associated with it.

In this paper we consider a simple asymmetric self-similar graph which does not satisfy the symmetry condition of [7]. In $\S 1.2$ we define a sequence of graphs $\left(G_{n}\right)_{n \in \mathbb{N}}$, which can be used to define a self-similar graph $G_{\infty}$ as for the self-similar graphs in [7]. 
In $\S 2$ we show that, for this example, a property similar to spectral decimation exists, in which (1.1) is replaced by

$$
2(1-\lambda)^{2}=f(\mu)
$$

where $f(\mu)$ is a quartic polynomial. Again there is an exceptional set of values of $\mu$ for which the relationship does not necessarily hold. This is proved in Theorems 2.1 and 2.2.

Another common spectral property of self-similar graphs and related fractals is that there are many eigenvalues of the Laplacian with high multiplicity and DirichletNeumann eigenfunctions, i.e. eigenfunctions which are zero on the boundary. In $[\mathbf{9}]$ it is shown that the eigenvalues with Dirichlet-Neumann eigenfunctions dominate the spectrum in a large class of cases, that of the nested fractals introduced in [5]. In [6] a similar result is shown for two-point self-similar graphs, a class which includes the example in this paper.

In $\S 3$ we calculate the number of linearly independent eigenfunctions of the Laplacian which are Dirichlet-Neumann or non-Dirichlet-Neumann. In $\S 4$, we use the results of $\S \S 2$ and $\S 3$ to describe the spectra of the graphs in the self-similar sequence of finite graphs used in the construction of our graph. This is stated in Theorem 4.1, which gives a complete description of the spectrum, including the multiplicity of the eigenvalues and which eigenvalues are associated with Dirichlet-Neumann and non-Dirichlet-Neumann eigenfunctions.

An example of a self-similar graph which does not satisfy the symmetry conditions of [7], and for which spectral decimation appears not to apply, is associated with the pentagasket, as described in [1] , in which numerical approximations for eigenvalues and eigenvectors are obtained, and some theoretical results are obtained that show how to construct eigenspaces of high multiplicity.

A more complicated method, using a rational map on a projective variety rather than on $\mathbb{R}$, which works for a larger class of self-similar graphs than that in [7] including some for which spectral decimation does not apply, is described in [10]. However, our graph does not meet all the conditions described in $\S 1.1 .1$ of $[\mathbf{1 0}]$.

\subsection{The graph}

In this section we define a self-similar sequence of finite graphs $\left(G_{n}\right)_{n \in \mathbb{N}}$.

We label the vertex sets and edge sets of a graph $G$ by $V(G)$ and $E(G)$, respectively, and, for a vertex $i \in V\left(G_{n}\right)$, we define $E_{i}^{(n)}$ to be the set of edges of $G_{n}$ connected to $i$.

We start with $G_{0}$, a single edge between two vertices 1 and 2 , and proceed inductively, constructing $G_{n+1}$ from $G_{n}$. Our graphs will be defined in such a way that $V\left(G_{n-1}\right) \subseteq V\left(G_{n}\right)$.

To construct $G_{n+1}$, we assume as an induction hypothesis that, if $n \geqslant 1$, the graph $G_{n}$ is bipartite with the two parts being $V\left(G_{n-1}\right)$ and $V\left(G_{n}\right) \backslash V\left(G_{n-1}\right)$, and hence that each edge $e \in E\left(G_{n}\right)$ can be thought of as connecting two vertices $i(e)$ and $j(e)$, defined so that $i(e) \in V\left(G_{n-1}\right)$ and $j(e) \in V\left(G_{n}\right) \backslash V\left(G_{n-1}\right)$. To deal with the special case $G_{0}$, we set $i\left(e_{0}\right)=1$ and $j\left(e_{0}\right)=2$ for its single edge $e_{0}$.

For each $e \in E\left(G_{n}\right)$ we introduce a new vertex, which we label $k(e)$, and we let the vertex set $V\left(G_{n+1}\right)$ of $G_{n+1}$ be the union of $V\left(G_{n}\right)$ with the set of new vertices 

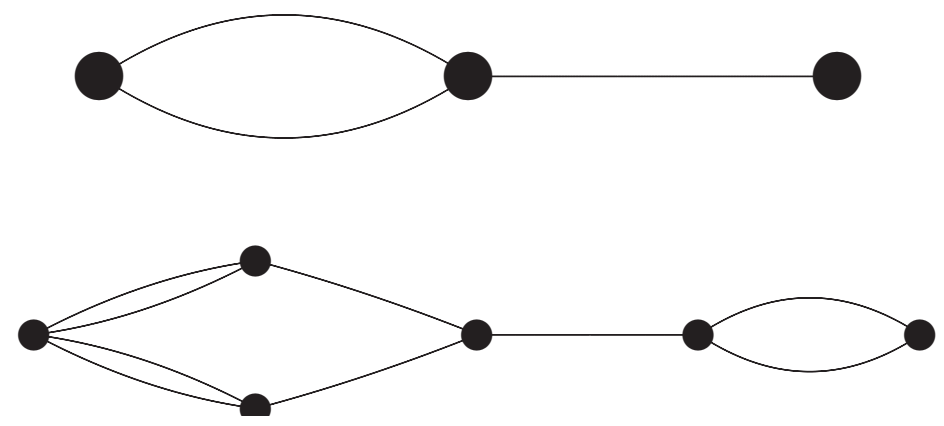

Figure 1. The graphs $G_{1}$ and $G_{2}$.

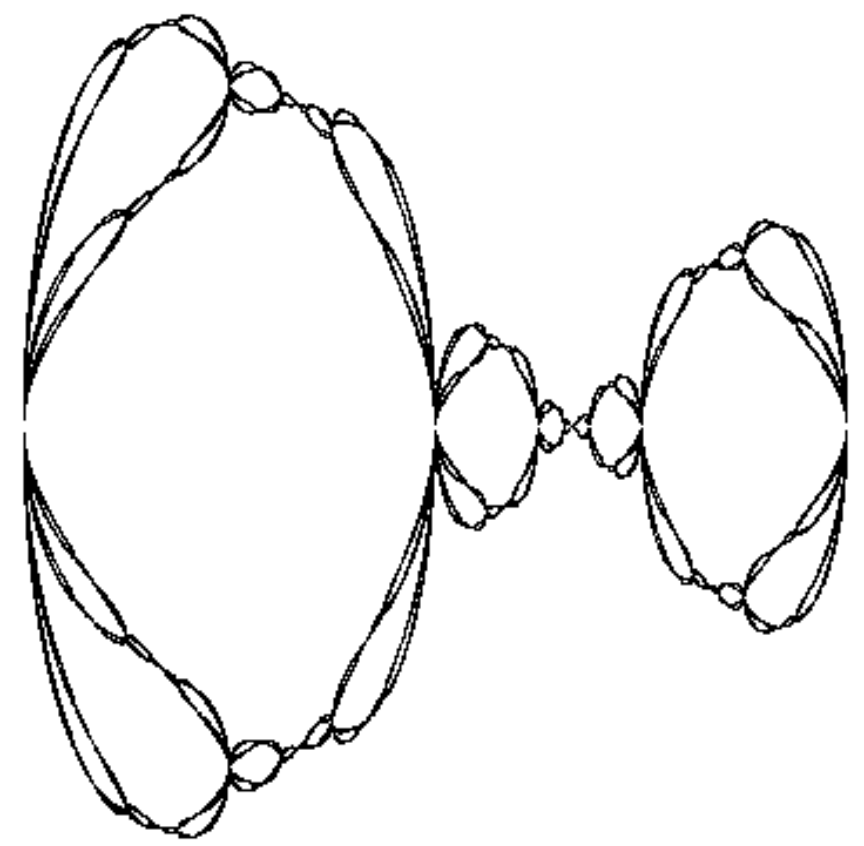

Figure 2. A later stage of the construction.

$\left\{k(e), e \in E\left(G_{n}\right)\right\}$. We then define $E\left(G_{n+1}\right)$ to consist of, for each $e \in E\left(G_{n}\right)$, two edges connecting $k(e)$ with $i(e)$ and one edge connecting $k(e)$ with $j(e)$. This ensures that the new graph $G_{n+1}$ is bipartite with the two parts being $V\left(G_{n}\right)$ and $V\left(G_{n+1}\right) \backslash V\left(G_{n-1}\right)$, so that we can continue the construction inductively.

Figure 1 shows $G_{1}$ and $G_{2}$, and Figure 2 shows a later stage of the construction, generated using MAPLE.

The same sequence of graphs can be obtained by using the framework of Definition 5.2 of [7], with the model graph being identical to $G_{1}$ above, but with conditions on the orientation to deal with the asymmetry.

It can also be obtained by a variation on the framework of $\S 1.1 .1$ of $[\mathbf{1 0}]$. In that 
framework, the sequence of graphs is obtained from a basic cell $F=\left\{1, \ldots, N_{0}\right\}$, for some $N_{0}$, and an equivalence relation $\mathcal{R}$ defined on $\{1, \ldots, N\} \times F$, where $N$ is the number of cells and satisfies $N \geqslant N_{0}$. In our case, $N_{0}=2$ and $N=3$, and our model graph $G_{1}$ can be defined by an equivalence relation $\mathcal{R}$ on $\{1,2,3\} \times\{1,2\}$ with three equivalence classes $\{(1,1),(3,1)\},\{(1,2),(2,2),(3,2)\}$ and the singleton $\{(2,1)\}$. However, in [10] the equivalence relation $\mathcal{R}$ is required to satisfy three conditions, one of which is that the equivalence class of $(i, i)$ for $1 \leqslant i \leqslant N_{0}$ should be a singleton, and our equivalence relation does not satisfy this condition, although it does satisfy the other two. As a result of this, the definitions of the equivalence relations $\mathcal{R}_{\langle\infty\rangle}$ and $\mathcal{R}_{\langle n\rangle}$, used in [10] to define the infinite graph and its subsets, need to be modified to deal with the more complicated behaviour of the boundary points.

When $1 \leqslant m<n$, the graph $G_{n}$ contains $3^{n-m}$ subgraphs isomorphic to $G_{m}$. We will call these subgraphs $m$-cells. Using this, we can define a sequence $\left(\tilde{G}_{n}\right)_{n \in \mathbb{N}}$ such that $\tilde{G}_{n}$ is isomorphic to $G_{n}$ and $\tilde{G}_{m}$ is a subgraph of $\tilde{G}_{n}$ for $m<n$. We then define the infinite graph $G_{\infty}=\bigcup_{n=0}^{\infty} \tilde{G}_{n}$. This is analogous to Definition 5.5 of [7].

We define maps $f_{i}: V\left(G_{n-1}\right) \rightarrow V\left(G_{n}\right), i=1,2,3$, mapping each vertex of $G_{n-1}$ to the corresponding vertex in each $(n-1)$-cell. We will label these so that $f_{1}$ and $f_{2}$ correspond to the two parallel cells.

We note that this graph is similar to that described in $[\mathbf{3}]$, although in the context of that paper the orientation of the cells is not important.

\subsection{The Laplacian}

There are a number of different definitions of the Laplacian of a graph. The definition of the graph Laplacian used in $[7]$ is the generator matrix of a continuous-time random walk on the graph, while in [2] a related symmetric matrix is used. However, the eigenvalues of the different definitions differ by at most a simple transformation.

For convenience in describing the eigenfunctions, we use the following definition of the Laplacian: the Laplacian $\mathcal{L}_{G}$ of a graph $G$ (which may have multiple edges but with no loops) is a $|V(G)| \times|V(G)|$ matrix with, for a vertex $i \in V(G), \mathcal{L}_{G}(i, i)=1$, and, for $i, j \in V(G)$ with $i \neq j, \mathcal{L}_{G}(i, j)=-e_{i, j} / \delta_{i}$, where $e_{i, j}$ is the number of edges linking $i$ and $j$ in $G$ and $\delta_{i}$ is the degree of vertex $i$ in $G$. This gives the same eigenvalues as the symmetric Laplacian described in $[\mathbf{2}]$, and the eigenfunctions are the 'harmonic eigenfunctions' described in [2]. Our definition of the graph Laplacian differs from that in [7] only in that the sign of each entry (and hence of the eigenvalues) is reversed.

\section{The relationship between the eigenvalues}

We set $f(\mu)=9(\mu-1)^{4}-9(\mu-1)^{2}+2$, so that (1.2) becomes

$$
2(1-\lambda)^{2}=9(\mu-1)^{4}-9(\mu-1)^{2}+2 .
$$

We first show how to construct eigenvalues $\mu$ of $\mathcal{L}_{G_{n+1}}$ from eigenvalues $\lambda$ of $\mathcal{L}_{G_{n}}$ when $\lambda \notin\{0,1,2\}$. 
Given $\lambda$ and $\mu$, we set

$$
\gamma=\frac{3(\mu-1)^{2}-2}{1-\lambda}
$$

Theorem 2.1. Given an eigenfunction $x$ of $\mathcal{L}_{G_{n}}$ with eigenvalue $\lambda \notin\{0,1,2\}$, we can do the following.

(i) Solve (2.1) for $\mu$ to obtain four roots.

(ii) For each possible $\mu$, set $\gamma$ using (2.2).

(iii) Then define $x^{\prime}$ by

$$
x_{i}^{\prime}= \begin{cases}x_{i} & i \in V\left(G_{n-1}\right), \\ \gamma x_{i} & i \in V\left(G_{n}\right) \backslash V\left(G_{n-1}\right),\end{cases}
$$

and, for a vertex $k=k(e) \in V\left(G_{n+1}\right) \backslash V\left(G_{n}\right)$, we set

$$
x_{k}^{\prime}=\frac{2 x_{i(e)}}{3(1-\mu)}+\frac{\gamma x_{j(e)}}{3(1-\mu)} .
$$

Then $x^{\prime}$ is an eigenfunction of $\mathcal{L}_{G_{n+1}}$ with eigenvalue $\mu$.

Proof. To check this, we just calculate $\mathcal{L}_{G_{n+1}} x^{\prime}$. For $i \in V\left(G_{n-1}\right)$,

$$
\begin{aligned}
\left(\mathcal{L}_{G_{n+1}} x^{\prime}\right)_{i} & =x_{i}+\frac{1}{\delta_{i}^{(n)}} \sum_{e \in E_{i}^{(n)}}\left(\frac{2 x_{i}}{3(\mu-1)}+\frac{\gamma x_{j(e)}}{3(\mu-1)}\right) \\
& =x_{i}+\frac{2 x_{i}}{3(\mu-1)}+\frac{\gamma x_{i}(1-\lambda)}{3(\mu-1)} \\
& =x_{i}\left(\frac{3(\mu-1)+2+3(\mu-1)^{2}-2}{3(\mu-1)}\right) \\
& =\mu x_{i}=\mu x_{i}^{\prime} .
\end{aligned}
$$

For $j \in V\left(G_{n}\right) \backslash V\left(G_{n-1}\right)$,

$$
\begin{aligned}
\left(\mathcal{L}_{G_{n+1}} x^{\prime}\right)_{j} & =\gamma x_{j}+\frac{1}{\delta_{j}^{(n)}} \sum_{e \in E_{j}^{(n)}}\left(\frac{2 x_{i(e)}}{3(\mu-1)}+\frac{\gamma x_{j}}{3(\mu-1)}\right) \\
& =\gamma x_{j}+\frac{\gamma x_{j}}{3(\mu-1)}+\frac{2 x_{j}(1-\lambda)}{3(\mu-1)} \\
& =x_{j}\left(\frac{3(\mu-1) \gamma+\gamma+2(1-\lambda)}{3(\mu-1)}\right) .
\end{aligned}
$$

Using (2.1) and (2.2),

$$
\begin{aligned}
2(1-\lambda) & =\frac{9(\mu-1)^{4}-9(\mu-1)^{2}+2}{1-\lambda} \\
& =\frac{\left(3(\mu-1)^{2}-1\right)\left(3(\mu-1)^{2}-2\right)}{1-\lambda} \\
& =\left(3(\mu-1)^{2}-1\right) \gamma,
\end{aligned}
$$


and so

$$
\begin{aligned}
\left(\mathcal{L}_{G_{n+1}} x^{\prime}\right)_{j} & =x_{j} \gamma\left(\frac{3(\mu-1)+1+3(\mu-1)^{2}-1}{3(\mu-1)}\right) \\
& =\mu \gamma x_{j} \\
& =\mu x_{j}^{\prime},
\end{aligned}
$$

and, finally, for $k \in j \in V\left(G_{n}\right) \backslash V\left(G_{n-1}\right)$, which satisfies $k=k(e)$ for some edge $e$ of $G_{n}$, we have

$$
\begin{aligned}
\left(\mathcal{L}_{G_{n+1}} x^{\prime}\right)_{k} & =x_{k}^{\prime}-\frac{2}{3} x_{i}^{\prime}(e)-\frac{1}{3} x_{j}^{\prime}(e) \\
& =\left(\frac{1}{1-\mu}-1\right)\left(\frac{2}{3} x_{i}+\frac{1}{3} \gamma x_{j}\right) \\
& =\mu\left(\frac{2 x_{i}}{3(1-\mu)}+\frac{\gamma x_{j}}{3(1-\mu)}\right) \\
& =\mu x_{k}^{\prime},
\end{aligned}
$$

so $x^{\prime}$ is indeed an eigenfunction of $\mathcal{L}_{G_{n+1}}$ with eigenvalue $\mu$.

Theorem 2.2. If

$$
\mu \notin\left\{1,1+\sqrt{\frac{2}{3}}, 1-\sqrt{\frac{2}{3}}, 1+\sqrt{\frac{1}{3}}, 1-\sqrt{\frac{1}{3}}\right\}
$$

and $\lambda$ and $\mu$ satisfy (2.1), then $\mu$ is an eigenvalue of $G_{n+1}$ if and only if $\lambda$ is an eigenvalue of $G_{n}$, with the same multiplicity.

Proof. If we have an eigenfunction $x^{\prime}$ of $\mathcal{L}_{G_{n+1}}$ with eigenvalue $\mu \neq 1$, then, for each edge $e$ of $G_{n+1}$,

$$
x_{k(e)}^{\prime}=\frac{2 x_{i(e)}^{\prime}}{3(1-\mu)}+\frac{x_{j(e)}^{\prime}}{3(1-\mu)},
$$

so that, for each $i \in V\left(G_{n-1}\right)$,

$$
\begin{aligned}
x_{i}^{\prime}(1-\mu) & =\frac{1}{\delta_{i}^{(n)}} \sum_{e \in E_{i}^{(n)}}\left(\frac{2 x_{i}^{\prime}}{3(1-\mu)}+\frac{x_{j(e)}^{\prime}}{3(1-\mu)}\right) \\
& =\frac{2 x_{i}^{\prime}}{3(1-\mu)}+\frac{1}{\delta_{i}^{(n)}} \sum_{e \in E_{i}^{(n)}} \frac{x_{j(e)}^{\prime}}{3(1-\mu)},
\end{aligned}
$$

giving

$$
x_{i}^{\prime}\left(3(1-\mu)^{2}-2\right)=\frac{1}{\delta_{i}^{(n)}} \sum_{e \in E_{i}^{(n)}} x_{j(e)}^{\prime} .
$$


Similarly, for $j \in V\left(G_{n}\right) \backslash V\left(G_{n-1}\right)$,

$$
\begin{aligned}
x_{j}^{\prime}(1-\mu) & =\frac{1}{\delta_{j}^{(n)}} \sum_{e \in E_{j}^{(n)}}\left(\frac{x_{j}^{\prime}}{3(1-\mu)}+\frac{2 x_{i(e)}^{\prime}}{3(1-\mu)}\right) \\
& =\frac{x_{j}^{\prime}}{3(1-\mu)}+\frac{1}{\delta_{j}^{(n)}} \sum_{e \in E_{j}^{(n)}} \frac{2 x_{i(e)}^{\prime}}{3(1-\mu)},
\end{aligned}
$$

giving

$$
x_{j}^{\prime}\left(3(1-\mu)^{2}-1\right)=\frac{1}{\delta_{j}^{(n)}} \sum_{e \in E_{j}^{(n)}} x_{i(e)}^{\prime} .
$$

By the conditions of the theorem, $(1-\mu)^{2} \neq \frac{2}{3}$. Then (2.5) implies that, for any $\lambda$,

$$
x_{i}^{\prime}(1-\lambda)=\frac{1}{\delta_{i}^{(n)}} \sum_{e \in E_{i}^{(n)}} \frac{1-\lambda}{3(1-\mu)^{2}-2} x_{j(e)}^{\prime},
$$

while (2.6) gives, if $\lambda \neq 1$,

$$
\frac{1-\lambda}{3(1-\mu)^{2}-2 x_{j}^{\prime}} \frac{\left(3(1-\mu)^{2}-2\right)\left(3(1-\mu)^{2}-1\right)}{2(1-\lambda)}=\frac{1}{\delta_{j}^{(n)}} \sum_{e \in E_{j}^{(n)}} x_{i(e)}^{\prime} .
$$

Then, if we set $x_{i}=x_{i}^{\prime}$ for $i \in V\left(G_{n-1}\right)$ and

$$
x_{j}=x_{j}^{\prime} \frac{1-\lambda}{3(1-\mu)^{2}-2},
$$

(2.7) and (2.8) become

$$
x_{i}(1-\lambda)=\frac{1}{\delta_{i}^{(n)}} \sum_{e \in E_{i}^{(n)}} x_{j(e)}
$$

and

$$
x_{j} \frac{\left(3(1-\mu)^{2}-2\right)\left(3(1-\mu)^{2}-1\right)}{2(1-\lambda)}=\frac{1}{\delta_{j}^{(n)}} \sum_{e \in E_{j}^{(n)}} x_{i(e)},
$$

which imply that $x$ is an eigenfunction of $\mathcal{L}_{G_{n}}$ with eigenvalue $\lambda$ if

$$
(1-\lambda)=\frac{\left(3(1-\mu)^{2}-2\right)\left(3(1-\mu)^{2}-1\right)}{2(1-\lambda)},
$$

which is equivalent to the quartic (2.1).

This eigenfunction can be degenerate only if $1-\lambda=0$, i.e. if either $(1-\mu)^{2}=\frac{1}{3}$ or $(1-\mu)^{2}=\frac{2}{3}$. 
The set

$$
\left\{1,1+\sqrt{\frac{2}{3}}, 1-\sqrt{\frac{2}{3}}, 1+\sqrt{\frac{1}{3}}, 1-\sqrt{\frac{1}{3}}\right\}
$$

of values of $\mu$ where Theorem 2.2 does not apply plays a similar role to that of the exceptional set in [7].

We note that the eigenvalues $\lambda$ and $2-\lambda$ produce the same values of $\mu$, with the same eigenfunctions. This is related to the bipartite nature of the graph; in fact, if $x$ is an eigenfunction with eigenvalue $\lambda$, then, following [2], we can obtain an eigenfunction with eigenvalue $2-\lambda$ by simply changing the sign of $x$ on $V\left(G_{n}\right) \backslash V\left(G_{n-1}\right)$. These two eigenfunctions will then produce the same new eigenfunction using the above construction.

We now consider the cases when Theorems 2.1 and 2.2 do not apply, i.e. when $\lambda \in$ $\{0,1,2\}$ or

$$
\mu \in\left\{1,1+\sqrt{\frac{2}{3}}, 1-\sqrt{\frac{2}{3}}, 1+\sqrt{\frac{1}{3}}, 1-\sqrt{\frac{1}{3}}\right\} .
$$

We note that if $\mu=1$ and $\lambda$ and $\mu$ satisfy (2.1), then $\lambda=0$ or 2 .

When $\lambda=1$ and $x_{i} \neq 0$ for some $i \in V\left(G_{n-1}\right)$, we use the same method, but with $\gamma=0$ and the quartic (2.1) replaced by

$$
(\mu-1)^{2}=\frac{2}{3} .
$$

We cannot use this method if $x_{i}=0$ for all $i \in V\left(G_{n-1}\right)$, because the constructed eigenfunction would be zero everywhere.

However, in the case where $\lambda=1$ and $x_{i}=0$ for all $i \in V\left(G_{n-1}\right)$, we can construct an eigenfunction $x^{\prime}$ by setting $x_{i}^{\prime}=0$ for $i \in V\left(G_{n-1}\right)$, and $x_{i}^{\prime}=x_{i}$ for $i \in V\left(G_{n}\right) \backslash V\left(G_{n-1}\right)$. This gives eigenvalues $\mu$ with

$$
(\mu-1)^{2}=\frac{1}{3}
$$

using similar methods to those above.

\section{Dirichlet-Neumann and non-Dirichlet-Neumann eigenfunctions}

The graph $G_{n}$ has $v_{n}$ vertices and $e_{n}$ edges where $v_{0}=2, e_{0}=1$ and $e_{n}=3 e_{n-1}$, $v_{n}=v_{n-1}+e_{n-1}$. Hence $e_{n}=3^{n}$ and $v_{n}=\frac{1}{2}\left(3^{n}+3\right)$.

The following lemma provides a means of constructing Dirichlet-Neumann eigenfunctions, which are zero on the two boundary vertices 1 and 2 .

Lemma 3.1. Let $\Gamma_{0}$ be a connected graph with $m$ vertices, including distinguished endpoints 1 and 2, and let $\Gamma$ be the graph formed by defining $\Gamma_{1}$ and $\Gamma_{2}$ to be two identical copies of $\Gamma_{0}$ and connecting them in parallel by identifying their endpoints. Then the Laplacian of $\Gamma$ has $m-2$ linearly independent eigenfunctions which are zero on the endpoints.

The associated eigenvalues are the eigenvalues of the Laplacian $\mathcal{L}_{\Gamma}$ restricted to the set $\{2 j: 2 \leqslant j \leqslant m-1\}$ of vertices in $\Gamma_{2}$.

Proof. We label the vertices of $\Gamma_{0}, 1,2, \ldots, m$. Then we label the vertices in $\Gamma$ so that, for $j \geqslant 3$, vertex $j$ in $\Gamma_{0}$ corresponds to vertex $2 j-3$ in $\Gamma_{1}$ and vertex $2 j-2$ in $\Gamma_{2}$. 
Now consider the Laplacian $\mathcal{L}_{\Gamma}$. For $2 \leqslant j, k \leqslant m-1$ we have

$$
\begin{aligned}
\mathcal{L}_{\Gamma}(1,2 j-1) & =\mathcal{L}_{\Gamma}(1,2 j), \\
\mathcal{L}_{\Gamma}(2,2 j-1) & =\mathcal{L}_{\Gamma}(2,2 j), \\
\mathcal{L}_{\Gamma}(2 j-1,2 k-1) & =\mathcal{L}_{\Gamma}(2 j, 2 k), \\
\mathcal{L}_{\Gamma}(2 j-1,2 k) & =\mathcal{L}_{\Gamma}(2 j, 2 k-1)=0
\end{aligned}
$$

and consider functions $x$ satisfying

$$
\begin{gathered}
x(1)=x(2)=0, \\
x(2 j-1)=-x(2 j) \quad \text { for } 2 \leqslant j \leqslant m-1 .
\end{gathered}
$$

Now

$$
\left(\mathcal{L}_{\Gamma} x\right)(1)=\sum_{j=2}^{m-1}\left(\mathcal{L}_{\Gamma}(1,2 j-1) x(2 j-1)+\mathcal{L}_{\Gamma}(1,2 j) x(2 j)\right)=0
$$

and, similarly, $\left(\mathcal{L}_{\Gamma} x\right)(2)=0$, while

$$
\begin{aligned}
\left(\mathcal{L}_{\Gamma} x\right)(2 j-1) & =\sum_{k=2}^{m-1} \mathcal{L}_{\Gamma}(2 j-1,2 k-1) x(2 k-1) \\
& =-\sum_{k=2}^{m-1} \mathcal{L}_{\Gamma}(2 j, 2 k) x(2 k)=\left(\mathcal{L}_{\Gamma} x\right)(2 j) .
\end{aligned}
$$

So the Laplacian $\mathcal{L}_{\Gamma}$ preserves vectors of this form, which form a vector space of dimension $m-2$, and it acts on them in a similar way to the Laplacian restricted to the interior vertices of $\Gamma_{0}$. As the Laplacian is symmetric, there are $m-2$ linearly independent Dirichlet-Neumann eigenfunctions of the Laplacian.

If $G_{n}$ contains a subgraph $\Gamma$ of this form, where the vertices of $\Gamma$ other than the endpoints have no edges linking them to $G_{n} \backslash \Gamma$, then we can take one of the eigenfunctions $x$ on $\Gamma$ constructed by the above lemma and extend it to an eigenfunction $\tilde{x}$ on $G_{n}$ by setting

$$
\tilde{x}(v)= \begin{cases}x(v) & \text { for } v \in V(\Gamma), \\ 0 & \text { otherwise. }\end{cases}
$$

If neither of the endpoints 1 or 2 is in the interior of the subgraph $\Gamma$, then this $\tilde{x}$ will be Dirichlet-Neumann.

Proposition 3.2. The graph $G_{n}$ has at least $\frac{1}{2}\left(3^{n}+3\right)-2^{n}-1$ linearly independent Dirichlet-Neumann eigenvalues.

Proof. The model graph contains parallel edges, so $G_{n}$ contains a subgraph consisting of two copies of $G_{n-1}$ with their boundary points identified as in Lemma 3.1. This gives $v_{n-1}-2$ eigenfunctions. For each eigenfunction $x$ obtained thus, we have $x\left(f_{1}(v)\right)=$ $-x\left(f_{2}(v)\right)$ and $x\left(f_{3}(v)\right)=0$ for each $v \in V\left(G_{n-1}\right)$. 
Furthermore, given a Dirichlet-Neumann eigenfunction $x$ of $G_{n-1}$, we can obtain three Dirichlet-Neumann eigenfunctions $x_{1}, x_{2}, x_{3}$ of $G_{n}$ by extending them from $(n-1)$-cells to the whole graph, i.e.

$$
x_{i}\left(f_{j}(v)\right)=\delta_{i j} x(v) \quad \text { for all } v \in V\left(G_{n-1}\right), \quad i, j=1,2,3 .
$$

However, we only obtain two linearly independent eigenfunctions, because the linear combination $x_{1}-x_{2}$ is of the form obtained using Lemma 3.1.

Hence, if $l_{n}$ is the number of Dirichlet-Neumann eigenvalues constructed by these methods, it satisfies

$$
l_{n}=2 l_{n}+\frac{1}{2}\left(3^{n-1}+3\right)-2
$$

and $l_{2}=1$, which gives the result.

Let the total number of Dirichlet-Neumann eigenfunctions of $G_{n}$ be $l_{n}+\hat{l}_{n}$, so that $\hat{l}_{n}$ is the number of eigenfunctions that are not constructed by the methods of Proposition 3.2 .

For this graph, we can also describe a set of non-Dirichlet-Neumann eigenfunctions.

Proposition 3.3. The graph $G_{n}$ has $2^{n}+1$ linearly independent eigenfunctions which are not Dirichlet-Neumann.

Proof. We consider the set of functions $x$ on $V\left(G_{1}\right)$ which are zero on the central vertex of $V\left(G_{1}\right)$, i.e. they satisfy $x(3)=0$ and, for each $v \in V\left(G_{n-1}\right), x\left(f_{1}(v)\right)=$ $x\left(f_{2}(v)\right)=\frac{1}{2} x\left(f_{3}(v)\right)$; these form a $\left(v_{n-1}-1\right)$-dimensional subspace. This is preserved by the Laplacian of $G_{n}$, so we can find $v_{n-1}-1=\frac{1}{2}\left(3^{n-1}+1\right)$ linearly independent eigenfunctions satisfying these properties.

To exclude those which are Dirichlet-Neumann, this is equivalent to the condition that $x(1)=x(2)=0$. So a Dirichlet-Neumann eigenfunction satisfying the above conditions reduces to a Dirichlet-Neumann eigenfunction on each $(n-1)$-cell. Hence there are $\frac{1}{2}\left(3^{n-1}+1\right)-l_{n-1}-\hat{l}_{n-1}=2^{n-1}-\hat{l}_{n-1}$ non-Dirichlet-Neumann eigenfunctions satisfying the above conditions.

Now, given any eigenfunction $x$ of $G_{n-1}$, we can extend it to an eigenfunction $x^{\prime}$ of $G_{n}$ by setting $x^{\prime}(1)=\sqrt{2} x(1), x^{\prime}(2)=x(1), x^{\prime}(3)=\sqrt{3} x(2), x^{\prime}\left(f_{1}(v)\right)=x^{\prime}\left(f_{2}(v)\right)=$ $x^{\prime}\left(f_{3}(v)\right)=x(v)$ for $v \geqslant 3$, from the structure of the graph. This means that each of the eigenfunctions constructed in Proposition 3.3 for $G_{n-1}$ can be extended to a nonDirichlet-Neumann eigenfunction of $G_{n}$, which will be linearly independent of those already found (because $x^{\prime}(3) \neq 0$ ). Inductively, this also applies to those constructed for $G_{n-m}, m>1$.

The graphs are bipartite, so eigenfunctions (which are zero nowhere) for eigenvalues 0 and 2 exist as described in [2].

Hence the total number of linearly independent non-Dirichlet-Neumann eigenfunctions is at least $2^{n}+1-\sum_{m=0}^{n-1} \hat{l}_{n}$. But we know that there are exactly $\frac{1}{2}\left(3^{n}+3\right)$ linearly independent eigenfunctions. Hence

$$
\frac{1}{2}\left(3^{n}+3\right) \geqslant 2^{n}+1-\sum_{m=0}^{n-1} \hat{l}_{m}+\frac{1}{2}\left(3^{n}+3\right)-2^{n}-1+\hat{l}_{n}
$$


and so $\hat{l}_{n} \leqslant \sum_{m=0}^{n-1} \hat{l}_{m}$. But $\hat{l}_{m}=0$ for $m \leqslant 2$, and hence for all $m$.

Hence the eigenfunctions constructed are all that exist, and there are $2^{n}+1$ linearly independent non-Dirichlet-Neumann ones, the remainder being Dirichlet-Neumann.

\section{The spectra of the graphs}

We can now use the relationships between eigenvalues and the information on DirichletNeumann and non-Dirichlet-Neumann eigenfunctions to obtain a complete description of the spectra of the graphs $G_{n}$.

Theorem 4.1. Set

$$
\alpha_{1}^{(1)}=1, \quad \alpha_{1}^{(2)}=1-\sqrt{\frac{2}{3}} \quad \text { and } \quad \alpha_{2}^{(2)}=1+\sqrt{\frac{2}{3}} .
$$

We extend this to define

$$
\left\{\alpha_{i}^{(n)} ; 1 \leqslant i \leqslant 2^{n-1}\right\}
$$

to be the $2^{n-1}$ values $\mu$ satisfying the quartic (2.1), with $\lambda=\alpha_{j}^{(n-1)}$ for some $j$.

Similarly, set

$$
\beta_{1}^{(1)}=1, \quad \beta_{1}^{(2)}=1-\sqrt{\frac{1}{3}} \quad \text { and } \quad \beta_{2}^{(2)}=1+\sqrt{\frac{1}{3}} .
$$

We extend this to define

$$
\left\{\beta_{i}^{(n)} ; 1 \leqslant i \leqslant 2^{n-1}\right\}
$$

to be the $2^{n-1}$ values $\mu$ satisfying the quartic (2.1), with $\lambda=\beta_{j}^{(n-1)}$ for some $j$.

Then we have the following.

(a) If $n \geqslant m$, then $\mathcal{L}_{G_{n}}$ has a non-Dirichlet-Neumann eigenfunction with eigenvalue $\alpha_{i}^{(m)}, 1 \leqslant i \leqslant 2^{m-1}$. Together with eigenfunctions with eigenvalues 0 and 2 , this describes the non-Dirichlet-Neumann spectrum of $\mathcal{L}_{G_{n}}$.

(b) If $n \geqslant m+1$, then $\mathcal{L}_{G_{n}}$ has $\frac{1}{2}\left(3^{n-m}-1\right)$ linearly independent Dirichlet-Neumann eigenvalues with eigenvalue $\beta_{i}^{(m)}$, for $1 \leqslant i \leqslant 2^{m-1}$.

Proof. We note that $G_{1}$ has a non-Dirichlet-Neumann eigenfunction with eigenvalue 1. As described in the proof of Proposition 3.3, this can then be extended to give a non-Dirichlet-Neumann eigenfunction $x$ with eigenvalue 1 for each $G_{n}, n \geqslant 1$.

Because this eigenfunction is non-Dirichlet-Neumann, it has $x_{i} \neq 0$ for at least some $i \in V\left(G_{n-1}\right)$. Hence the construction of eigenfunctions of $G_{n+1}$ with eigenvalues $\mu$ satisfying $(1-\mu)^{2}=\frac{2}{3}$ produces non-degenerate eigenfunctions, which are also non-DirichletNeumann. So $G_{n+1}$ has non-Dirichlet-Neumann eigenfunctions with eigenvalues

$$
1 \pm \sqrt{\frac{2}{3}}
$$

We have already shown that $G_{n}$ has a non-Dirichlet-Neumann eigenfunction with eigenvalue $\alpha_{i}^{(2)}, 1 \leqslant i \leqslant 2^{n-1}$, for $n \geqslant 2$. Now, if $G_{n-1}$ has a non-Dirichlet-Neumann 
eigenfunction with eigenvalue $\alpha_{i}^{(m-1)}$ for each $1 \leqslant i \leqslant 2^{m-2}$, then the construction of eigenfunctions gives us a non-Dirichlet-Neumann eigenfunction of $G_{n}$ with eigenvalue $\alpha_{i}^{(m)}$ for each $1 \leqslant i \leqslant 2^{m-1}$. Using this inductively, we find that $G_{n}$ has a non-DirichletNeumann eigenfunction with eigenvalue $\alpha_{i}^{(m)}, 1 \leqslant i \leqslant 2^{m-1}$, for $n \geqslant m$.

Along with the eigenfunctions with eigenvalues 0 and 2 , this gives us all $2^{n}+1$ nonDirichlet-Neumann eigenfunctions from Proposition 3.3, and hence completes the proof of (a).

We now consider the Dirichlet-Neumann eigenfunctions. We note that $G_{2}$ has a Dirichlet-Neumann eigenfunction with eigenvalue 1 . Such an eigenfunction is zero on $V\left(G_{1}\right)$, so our main construction produces a degenerate eigenfunction. However, the alternative construction with $(1-\mu)^{2}=\frac{1}{3}$ does produce two eigenfunctions of $G_{3}$. Hence, as there are $\frac{1}{2}\left(3^{n}+3\right)-2^{n}-1$ Dirichlet-Neumann eigenfunctions of $G_{n}$, we can use the constructions to obtain $3^{n}+3-2^{n+1}-2$ Dirichlet-Neumann eigenfunctions, with eigenvalues other than 1 , of $G_{n+1}$.

We now show that, for $n \geqslant 2, G_{n}$ has $\frac{1}{2}\left(3^{n-1}-1\right)$ linearly independent DirichletNeumann eigenfunctions with eigenvalue 1 . This is the case for $n=2$. For each such eigenfunction of $G_{n}$, we can construct three eigenfunctions of $G_{n+1}$ using the methods in the proof of Proposition 3.2.

Assuming that $G_{n}$ has $\frac{1}{2}\left(3^{n}+3\right)-2^{n}-1$ linearly independent Dirichlet-Neumann eigenfunctions of which $\frac{1}{2}\left(3^{n-1}-1\right)$ have eigenvalue 1 , we have $\frac{1}{2}\left(3^{n}-3\right)$ linearly independent Dirichlet-Neumann eigenfunctions of $G_{n+1}$ with eigenvalue 1 , and $3^{n}+3-$ $2^{n+1}-2$ with other eigenvalues. However, we know from Proposition 3.2 that there are $\frac{1}{2}\left(3^{n+1}+3\right)-2^{n+1}-1$ in total, and

$$
\frac{1}{2}\left(3^{n}-3\right)+3^{n}+3-2^{n+1}-2=\frac{1}{2}\left(3^{n+1}+3\right)-2^{n+1}-2 .
$$

The one unexplained eigenfunction must also have eigenvalue 1 because, if it had eigenvalue $\lambda \neq 1$, we would also have an unexplained eigenfunction with eigenvalue $2-\lambda$. Hence we have $\frac{1}{2}\left(3^{n}-1\right)$ linearly independent Dirichlet-Neumann eigenfunctions of $G_{n+1}$ with eigenvalue 1 , giving the result by induction.

We know that, for $n \geqslant 2, G_{n}$ has $\frac{1}{2}\left(3^{n-1}-1\right)$ linearly independent Dirichlet-Neumann eigenfunctions with eigenvalue 1 . We now use our constructions $m$ times to show that, for $n \geqslant m+1, G_{n}$ has $\frac{1}{2}\left(3^{n-m}-1\right)$ linearly independent Dirichlet-Neumann eigenfunctions with eigenvalue $\beta_{i}^{(m)}$, for $1 \leqslant i \leqslant 2^{m-1}$. This completes the proof of (b).

\section{Reversing the orientation}

We remark that very similar results can be obtained if we reverse the orientation of the model graphs in the definitions of $\S 1.2$. Because of the asymmetry this gives a different self-similar sequence of graphs. Eigenvalues $\lambda$ and $\mu$ of Laplacians of successive members of the sequence are related by the same equation (2.1) when $\lambda \notin\{0,1,2\}$, but the two equations (2.9) and (2.10) for $\mu$ when $\lambda=1$ are reversed. This has the effect that, in Theorem 4.1, the roles of $\alpha_{i}^{(n)}$ and $\beta_{i}^{(n)}$ are reversed. 


\section{References}

1. B. Adams, S. A. Smith, R. S. Strichartz and A. Teplyaev, The spectrum of the Laplacian on the pentagasket, in Fractals in Graz 2001, Trends in Mathematics, pp. 1-24 (Birkhäuser, Basel, 2003).

2. F. R. K. Chung, Spectral graph theory, CBMS Regional Conference Series, no. 92 (American Mathematical Society, Providence, RI, 1997).

3. C. D. Essoh And J. Bellisard, Resistance and fluctuation of a fractal network of random resistors: a non-linear law of large numbers, J. Phys. A 22 (1989), 4537-4548.

4. M. Fukushima And T. Shima, On a spectral analysis for the Sierpiński gasket, Potent. Analysis 1 (1992), 1-35.

5. T. LindstrøM, Brownian motion on nested fractals, Memoirs of the American Mathematical Society, vol. 420 (American Mathematical Society, Providence, RI, 1990).

6. L. Malozemov and A. Teplyaev, Pure point spectrum of the Laplacians on fractal graphs, J. Funct. Analysis 129 (1994), 390-405.

7. L. Malozemov and A. Teplyaev, Self-similarity, operators and dynamics, Math. Phys. Analysis Geom. 6 (2003), 201-218.

8. R. Rammal and G. Toulouse, Random walks on fractal structures and percolation clusters, J. Phys. (Paris) Lett. 44 (1983), L13-L22.

9. C. Sавот, Pure point spectrum for the Laplacian on unbounded nested fractals, J. Funct. Analysis 173 (2000), 497-524.

10. C. SАвот, Spectral properties of self-similar lattices and iteration of rational maps, Mémoires de la Société Mathématique de France, vol. 92 (Société Mathématique de France, Paris, 2003).

11. T. SHImA, On eigenvalue problems for the random walks on the Sierpiński pre-gaskets, Jpn. J. Ind. Appl. Math. 8 (1991), 127-141.

12. T. ShimA, On eigenvalue problems for Laplacians on P.C.F. self-similar sets, Jpn. J. Ind. Appl. Math. 13 (1996), 1-23. 